\title{
Anti-Fibrillarin Antibody in African American Patients with Systemic Sclerosis: Immunogenetics, Clinical Features, and Survival Analysis
}

Roozbeh Sharif, MD ${ }^{1}$, Marvin J. Fritzler, MD, PhD $^{2}$, Maureen D. Mayes, MD, MPH ${ }^{1}$, Emilio B. Gonzalez, MD ${ }^{3}$, Terry A. McNearney, MD ${ }^{3,}{ }^{\star}$, Hilda Draeger, MD $^{4}$, Murray Baron, MD $^{5}$, the Canadian Scleroderma Research Group ${ }^{6}$, Daniel E. Furst, MD7 , Dinesh Khanna, MD, MS ${ }^{7}$, Deborah J. del Junco, PhD ${ }^{1}$, Jerry A. Molitor, MD, PhD $^{8}$, Elena Schiopu, MD ${ }^{9}$, Kristine Phillips, MD, PhD $^{9}$, James R. Seibold, $\mathbf{M D}^{9}$, Richard M. Silver ${ }^{10}$, Robert Simms, MD ${ }^{11}$, GENISOS Study Group ${ }^{12}$, Marilyn Perry, BSc ${ }^{1}$, Carlos Rojo, BSc ${ }^{1}$, Julio Charles, BSc ${ }^{1}$, Xiaodong Zhou, MD ${ }^{1}$, Sandeep K. Agarwal ${ }^{1}$, John D. Reveille ${ }^{1}$, Shervin Assassi, MD, MS ${ }^{1}$, and Frank C. Arnett, MD ${ }^{1}$

${ }^{1}$ Division of Rheumatology and Immunogenetics, University of Texas Health Science Center at Houston, TX

2 Department of Medicine, University of Calgary, Calgary, Canada

${ }^{3}$ Division of Rheumatology, University of Texas Medical Branch at Galveston, TX

4 Division of Rheumatology, University of Texas Health Science Center at San Antonio, TX

${ }^{5}$ Jewish General Hospital and McGill University, Montreal, Canada

Address for Correspondence and Reprint Request: Roozbeh Sharif, M.D. Post-doctoral fellow, The University of Texas-Health Science Center at Houston, 6431 Fannin St, MSB 5.261, Houston, TX 77030, roozbeh.sharif@uth.tmc.edu Phone: (713) 500-6298, Fax: (713) 500-0580

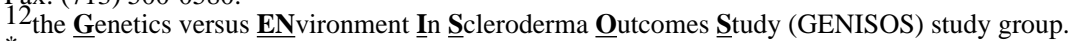

* Dr McNearney is currently employed at Eli Lilly and Company, Indianapolis, IN.

Roozbeh Sharif, MD: Postdoctoral Fellow at University of Texas Health Science Center at Houston, Houston, TX,

Marvin J. Fritzler, MD, PhD: Professor of Medicine at University of Calgary, Calgary, Canada,

Maureen D. Mayes, MD, MPH: Professor of Medicine at University of Texas Health Science Center at Houston, Houston, TX,

Emilio B. Gonzalez, MD: Professor of Medicine at University of Texas Medical Branch, Galveston, TX,

Terry A. McNearney, MD: Professor of Medicine at University of Texas Medical Branch, Galveston, TX, (currently employed at Eli Lilly and Company, Indianapolis, IN).

Hilda Draeger, MD: Assistant Professor of Medicine at University of Texas Health Science Center at San Antonio, San Antonio, TX,

Murray Baron, MD: Professor of Medicine at Jewish General Hospital, Director of Canadian Scleroderma Research Group

Daniel E. Furst, MD: Professor of Medicine at University of California at Los Angeles, Los Angeles, CA

Dinesh K. Khanna, MD, MS: Associate Professor of Medicine at University of California at Los Angeles, Los Angeles, CA

Deobrah J. del Junco, PhD: Director of Outcome Research Center for Translational Injury Research (CeTIR) at University of Texas

Health Science Center at Houston, Houston, TX,

Jerry A. Molitor, MD, PhD: Associate Professor of Medicine at University of Minnesota, Minneapolis, MN.

Elena Schiopu, MD: Assistant Professor of Medicine at University of Michigan, Ann Arbor, MI,

Kristine Phillips, MD, PhD: Assistant Professor of Medicine at University of Michigan, Ann Arbor, MI,

James R. Seibold, MD, Professor of Medicine at University of Michigan, Ann Arbor, MI, (currently at Scleroderma Research

Consultants, LLc, Avon, CT).

Richard M. Silver: Professor of Medicine and Pediatrics at Medical University of South Carolina, Charleston, SC,

Robert W. Simms, MD: Professor of Medicine at Boston University, Boston, MA,

Marilyn Perry, BSc: Senior Research Associate at University of Texas Health Science Center at Houston, Houston, TX,

Carlos Rojo, BSc; University of Texas Health Science Center at Houston, Houston, TX,

Julio Charles, BSc; Senior Research Associate at University of Texas Health Science Center at Houston, Houston, TX,

Xiadong Zhou, MD, Associate Professor of Medicine at University of Texas Health Science Center at Houston, Houston, TX,

Sandeep K. Agarwal: Associate Professor of Medicine at University of Texas Health Science Center at Houston, Houston, TX,

John D. Reveille: Professor of Medicine at University of Texas Health Science Center at Houston, Houston, TX,

Shervin Assassi, MD, MS: Assistant Professor of Medicine at University of Texas Health Science Center at Houston, Houston, TX,

Frank C. Arnett, MD: Professor of Medicine at University of Texas Health Science Center at Houston, Houston, TX. 
${ }^{6}$ The Canadian Scleroderma Research Group: Janet E Pope, Janet Markland, David Robinson D, Niall Jones, Nader Khalidi, Peter Docherty, Maysan Abu-Hakima, Sharon LeClercq, Evelyn Sutton, Douglas Smith, Jean-Pierre Mathieu, Alejandra Masetto, Elzbieta Kaminska, Sophie Ligier

7 Division of Rheumatology, Department of Medicine, David Geffen School of Medicine at UCLA, Los Angeles, CA

8 Division of Rheumatic and Autoimmune Diseases, University of Minnesota

${ }^{9}$ University of Michigan Scleroderma Program, Ann Arbor, MI

10 Division of Rheumatology and Immunology, Medical University of South Carolina, Charleston, NC

${ }^{11}$ Rheumatology Section, Department of Medicine, Boston University School of Medicine, MA

\section{Abstract}

Background-Anti-U3-RNP or anti-fibrillarin antibodies (AFA) are detected more frequently among African American (AA) patients with systemic sclerosis (SSc) compared to other ethnic groups and are associated with distinct clinical features. The current study examines the immunogenetic, clinical, and survival correlates of AFA in a large group of AA patients with SSc.

Methods-Overall, 278 AA SSc patients and 328 unaffected AA controls were enrolled from three North American cohorts. Clinical features, autoantibody profile, and HLA-class-II genotyping were captured. To compare the clinical manifestations, relevant clinical features were adjusted for disease duration. The Cox proportional hazards regression was used to determine the effect of AFA on survival.

Results-Fifty (18.5\%) AA patients had AFA. After Bonferroni correction, HLA-DRB1*08:04 was associated with AFA, compared to unaffected AA controls $(\mathrm{OR}=11.5, \mathrm{p}<0.0001)$ and AFA negative $\mathrm{SSc}$ patients $(\mathrm{OR}=5.2, \mathrm{p}=0.0002)$. AFA positive AA patients had younger age of disease onset, higher frequency of digital ulcers, diarrhea, pericarditis, higher Medsger Perivascular and lower Lung Severity Indices $(p=0.004, p=0.014, p=0.019, p=0.092, p=0.006$, and $p=0.016$, respectively). After adjustment for age at enrollment, AFA positive patients did not have different survival compared with patients without AFA $(p=0.493)$.

Conclusion-These findings demonstrate strong association between AFA and HLA$D R B I * 08: 04$ allele in AA patients with SSc. Moreover, AA SSc patients with AFA had younger age of onset, higher frequency of digital ulcers, pericarditis, and severe lower gastrointestinal involvement, but less severe lung involvement compared to AA patients without AFA. However, presence of AFA did not change survival.

\section{Keywords}

Scleroderma; GENISOS; anti-U3-RNP; digital ulcer; HLA DRB1; and Scleroderma Family Registry

\section{INTRODUCTION}

African American (AA) patients with systemic sclerosis (SSc; scleroderma) are reported to have a worse overall prognosis than Caucasians which may be reflected by a younger age of disease onset, higher frequency of diffuse cutaneous involvement, digital ulcers and pits, more severe lung involvement and younger age at onset of pulmonary artery hypertension $(\mathrm{PAH})(1-5)$. 
Anti-U3-RNP or anti-fibrillarin antibody (AFA) is directed against a $35 \mathrm{kD}$ protein component of a nucleolar ribonucleoprotein called fibrillarin, which is an early marker for the site of formation of nucleolus in dividing cells (6). The frequency of AFA differs across ethnic groups, ranging from zero in a large cohort of Italian patients with SSc (7) to 50\% in an African American SSc population (8). The higher prevalence of AFA in the sera of African American (AA) patients with SSc has been noted in several studies (9-13).

Previous studies have shown that HLA-DRB $* 08$ and $D Q B 1 * 03: 01$ are associated with AFA in African Americans (10;14). Clinically, SSc patients with AFA have been reported to have younger ages of disease onset, higher frequency of diffuse cutaneous involvement, pulmonary artery hypertension (PAH), SSc-associated musculoskeletal and cardiac involvement, and lower frequency of arthritis $(9-11 ; 15-17)$. However, there is a lack of large and robust studies on the immunogenetic associations, clinical manifestations, and survival effect of AFA in African American (AA) patients with SSc.

This study compared the HLA class-II alleles in AA SSc patients with AFA with ethnicgender matched unaffected controls and with SSc patients without AFA. In addition, we investigated the clinical features and survival effect of AFA in AA patients with SSc.

\section{MATERIALS AND METHODS}

\section{Study population}

Between 1985 and 2010, 3033 patients with SSc were enrolled in the following cohorts: (a)

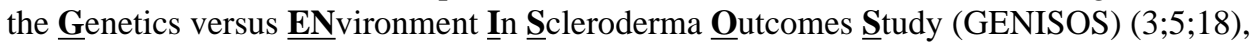
(b) the NIH/NIAMS Scleroderma Family Registry and DNA Repository (19), and (c) Division of Rheumatology at University of Texas Health Science Center at Houston (UTHSC-H) (10). Patients were included if they met the American College of Rheumatology (ACR; formerly, the American Rheumatism Association) classification criteria for SSc (20) or had at least three of the five CREST (calcinosis, Raynaud's phenomenon, esophageal dysmotility, sclerodactyly, and telangiectasias) features (21). We included all African American patients from these cohorts $(n=278)$. Patients enrolled in more than one of the above-mentioned cohorts were identified and duplicate entries were omitted. Furthermore, we enrolled 328 unaffected AA controls to determine any HLA class II allele associations with AFA. The unaffected AA individuals were volunteers with no personal or family history of SSc or other autoimmune disease by screening questionnaire. All enrolled study subjects (SSc patients and unaffected controls) provided written informed consent and the institutional review board of all participating institutions approved the study.

\section{Autoantibody profile and HLA class II allele genotyping}

All autoantibody determinations and HLA class II allele typing were conducted in the Division of Rheumatology at UTHSC-H Houston, TX and the Mitogen Advanced Diagnostics Laboratory, University of Calgary, Calgary, Canada. Anti-nuclear antibodies (ANA) and anti-centromere antibodies (ACA) were determined using indirect immunofluorescence with HEp-2 cells as the antigen substrate (Antibodies Inc., Davis, CA, USA). Passive immunodiffusion gels against calf thymus extract were used to examine sera for anti-topoisomerase-I (ATA; Scl-70), anti-Ro/SS-A, anti-La/SS-B, and anti-U1-RNP autoantibodies (INOVA Diagnostics, San Diego, California, USA). Anti-RNA polymerase III (RNAP III) was detected by enzyme-linked immunosorbent immunoassay (ELISA) kits (MBL Co. Ltd, Nagoya, Japan) and AFA were determined by a line immunoassay at a serum dilution of 1:1000 using purified recombinant fibrillarin protein (Euroline-WB: Euroimmun, Lubeck, Germany) in patients who had a positive ANA in anti-nucleolar pattern on the indirect immunofluorescence. 
As previously described (5;22), we genotyped HLA class II alleles (DRB1, DQA1, DQB1, and $D P B 1$ ) on extracted and purified genomic DNA. Furthermore, we examined the HLA class-II allele binding peptide by Propred MHC Class II Binding Peptide Prediction Server in order to predict binding peptides of human fibrillarin protein. This prediction is based on quantitative matrices derived from the literature $(23 ; 24)$.

\section{Clinical manifestation}

Age, gender, disease type (categorized as limited or diffuse cutaneous involvement at time of enrollment (21)), disease duration (calculated from the onset of the first non-Raynaud's phenomenon symptom attributable to SSc), and modified Rodnan Skin Score (MRSS (25)) were recorded.

To assess the severity of the individual organ system involvement, the Medsger severity indices $(13 ; 26)$ of eight organ systems were captured: peripheral vessels, skin, joints/ tendons, skeletal muscle, gastrointestinal (GI) tract, lung, heart, and kidney. However, these data were available only for the patients enrolled in the GENISOS cohort $(n=78)$. The presence of digital ulcers was determined based on the participating rheumatologist's clinical assessment. Arthritis was defined as presence of joint swelling and tenderness on physical examination not attributable to osteoarthritis, crystalline arthropathy, or trauma. A decrease in range of motion $>25 \%$ in at least one joint axis was defined as joint contracture. Dysphagia, diarrhea attributable to SSc, and history of SSc renal crisis were recorded. Electrocardiography and 2-dimensional echocardiography findings and/or presence of an auscultatory friction rub determined the presence of pericarditis or clinically significant pericardial effusion.

As previously described (18), pulmonary function tests were obtained at enrollment. Interstitial lung fibrosis was defined as chest radiographs showing fibrosis and/or a forced vital capacity (FVC) of less than $75 \%$ predicted value was recorded.

For the purpose of our review, pulmonary artery hypertension (PAH) was defined if the patient had (a) mean pulmonary artery pressure (mPAP) equal or higher than $25 \mathrm{~mm} \mathrm{Hg}$ on right heart catheterization, (b) right ventricular systolic pressure $\geq 40 \mathrm{mmHg}$ on 2dimensional echocardiography, or (c) the ratio of FVC \% predicted to diffusion capacity of carbon monoxide (DLCO) \% predicted $\geq 1.6$. Serum creatinine kinase (CK) levels were recorded and myositis was diagnosed if the patient had proximal muscle weakness with at least one of the following: elevated levels of CK, features of myositis on electromyography, and/or a characteristic muscle biopsy.

\section{Death search}

The vital status of the patients was determined through the National Death Index (NDI), at Centers for Disease Control and Prevention (CDCP), which provided data up until 2007. We then reviewed the Social Security Death Index (SSDI) to update our results as of August 2010. SSDI is an online death search tool that provides fatality reports based on observing death certificates and family confirmation. Those patients who were alive based on NDI report and no further records were found on SSDI were assumed to be alive.

\section{Statistical analysis}

Homozygosity for alleles at each of the tested HLA loci was not suggestive of recessive inheritance, regardless of whether the referent comparison group was disease-free controls or AFA-negative cases. Besides, there were too few homozygous subjects to distinguish between additive $v s$. dominant modes of inheritance, regardless of the referent. Therefore, a dominant mode of inheritance approach was used to compare the HLA association with 
AFA. Heterozygosity and homozygosity for a particular allele were both re-coded as ' 1 ' in a binary (zero or one) variable created for each specific HLA gene of interest. In other words, subjects negative for the gene on both of their alleles for the particular HLA locus were coded " 0 " for the gene on the new binary variable. Bonferroni correction for multiple comparisons was performed for HLA allelic analyses.

Moreover, age, gender, disease type, and duration between AFA positive and AFA negative patients was evaluated utilizing $\chi^{2}$ and student's t-test accordingly. SSc clinical manifestations might change over the course of disease. Therefore, logistic regression was used to adjust for disease duration as a possible confounding factor in clinical features and to examine the independent effect of AFA.

We utilized a Cox proportional hazards regression analysis to examine the association of AFA with survival. Besides, we investigated the potential association of relevant HLA classII with survival of the AA patients with SSc. The survival analysis was corrected for age at enrollment. Survival was calculated from the date of enrollment.

ATA and AFA are the two most common anti-nuclear antibodies among AA patients with SSc. We also compared the clinical features and survival of AA scleroderma patients with AFA $(n=50)$ to those with ATA $(n=61)$ in order to conduct our comparative analysis between more homogenous groups.

All the statistical analyses were performed with SAS Version 9.2 (SAS Institute Inc., Cary, NC) and STATA 11 (StataCorp, College Station, TX). The hypothesis testing was 2-sided with a $\mathrm{p} \leq 0.05$ significance level.

\section{RESULTS}

\section{Study population, disease, and autoantibody characteristics}

All 278 AA scleroderma patients from the three cohorts were included in the study. The mean age $( \pm \mathrm{SD})$ of patients at enrollment was $46.9(13.9)$ years and $237(85.3 \%)$ were female. At enrollment, 171 (61.5\%) AA patients with SSc were diagnosed with diffuse cutaneous involvement. Average disease duration $( \pm \mathrm{SD})$ was $6.0(6.5)$ years.

Anti-nuclear antibodies (ANA) on HEp-2 substrate were detected in $93.1 \%$ of AA SSc patients. ATA, RNAP-III, and AFA were present in $21.8 \%, 15.4 \%$, and $18.5 \%$ of patients, respectively (table 1).

\section{HLA class II allelic frequencies}

As illustrated in table 2, comparing the HLA class II allelic frequencies of AFA positive patients with 329 ethnically matched unaffected controls demonstrated that the HLA$D R B 1 * 08: 04$ allele was seen more frequently in AFA positive patients ( $47.6 \%$ vs. $6.4 \%$; odds ratio (OR): 11.52; 95\% confidence interval (CI): 5.43, 24.40; corrected $p<0.0001$ ). Two other alleles, which are located on the same haplotype $D Q A 1 * 04: 01$ and $D Q B 1 * 03: 01$, had similar patterns. However, the increased frequency of $D Q A 1 * 04: 01$ was not statistically significant.

Moreover, the frequency of HLA-DRBI*08: 04 in AFA positive patients also was higher in comparison to AA patients without AFA, even after correction for multiple comparisons (47.6\% vs. 14.9\%; OR: 5.21; CI: 2.44, 11.09; corrected $p=0.0002$ ). Both HLA $D Q A 1 * 04: 01$ and $D Q B 1 * 03: 01$ showed similar trends. However, neither of them remained significant after correction for multiple comparisons. 
HLA $D P B 1 * 01: 01$ was also seen more frequently among AFA positive AA patients compared to unaffected controls and SSc patients without AFA while HLA DRB1*11:01 seemed to be protective. HLA $D P B 1 * 01: 01$ and HLA $D R B 1 * 11: 01$ are not in linkage disequilibrium with HLA $D R B 1 * 08: 04$. However, the association of these two alleles with AFA did not withstand correction for multiple comparisons. The frequencies of all relevant HLA class II alleles in AA SSc patients and unaffected individuals are illustrated in supplement table 1 .

HLA DRB1*08:04 binding peptides-Using virtual matrix for HLA DRBI*08:04, at a threshold of $1 \%$ (the percentage of best scoring natural peptides), we identified four binding peptides (FRSKLAAAI, FRGRGRGGG, IHIKPGAKV and FVISIKANC) from the human fibrillarin protein that could serve as potential binding sites within the antigen binding groove.

\section{Clinical features}

AA SSc patients with AFA were younger at disease onset $(p=0.004)$ but gender, disease type, and duration were not significantly different between the two SSc groups. Table 3 illustrates the comparison of clinical manifestations between AFA positive and AFA negative AA patients with SSc.

After adjusting for disease duration, AA SSc patients with AFA had were 3.31 times more likely to have digital ulcers $(p=0.014)$. Diarrhea and pericarditis occurred more frequently in AFA positive AA SSc patients (OR 4.84; $p=0.019$ and OR: $2.45 ; p=0.092$, respectively) than AA patients without AFA. However, there was no difference between AFA positive and negative AA SSc patients in MRSS, dysphagia, pulmonary artery hypertension (PAH), SScassociated interstitial lung fibrosis, $\mathrm{FVC}$ and $\mathrm{D}_{\mathrm{L}} \mathrm{CO} \%$ predicted values, $\mathrm{SSc}$ renal crisis, myositis or muscle weakness, serum CK, joint contracture, or sicca symptoms.

Moreover, AFA positive patients had higher Medsger peripheral vascular severity index (regression coefficient (b): 0.79; CI: $0.27,1.30 ; p=0.003$ ), indicating more severe peripheral vascular involvement and lower Medsger lung severity index (b: -0.82 ; CI: $-1.50,-0.14$; $p=0.019$ ), indicating less severe lung involvement. The other Mesdger severity indices were not significantly different (table 3 ).

\section{Survival analysis}

At the time of analysis, $30 \%$ of AFA positive patients and 29.5\% of AFA negative patients were deceased (table 3). After correction for age at enrollment, AFA positive patients did not have different survival compared to AFA negative AA SSc patients (Hazard ratio=0.79, $p=0.493$ ). In addition, none of the relevant HLA class-II was predictor of mortality in AA patients with SSc (table 4).

\section{AFA Versus ATA among AA patients with SSc}

Although, the age at the onset of first non-Raynaud phenomenon symptom was not statistically different between two groups, the AA scleroderma patients with AFA had higher frequency of digital ulcer, lower GI tract involvement, pericarditis, and Medsger peripheral vascular severity index (Supplement 2). While AFA positive patients had lower Medsger lung severity index, higher FVC and DLCO \% predicted values, less cases of PAH. Despite, less severe lung disease, after adjusting for age of disease onset, AFA positive patients did not have better or worse survival compared to ATA positive (table 4). 


\section{DISCUSSION}

At a frequency of $18.5 \%$, AFA is the second most common anti-nuclear antibody among AA patients with SSc (second to ATA). The present report is the first study investigating the genetic associations, clinical manifestations, and survival impact of AFA in a large population of AA SSc patients.

Distinct HLA class-II allelic associations of SSc-specific autoantibodies in different ethnic groups have been demonstrated in several studies $(5 ; 10 ; 14 ; 27 ; 28)$. In a large sample of Caucasian patients, we have previously reported that the HLA $D R B 1 * 13: 02$,

$D Q B 1 * 06: 04 / 06: 05$ haplotype correlated with AFA (14). In the current study, we did not observe a similar pattern among AA patients with AFA. Our results indicated that HLA $D R B I * 08: 04$ is strongly associated with AFA in AA patients with SSc, compared to either unaffected individuals or AFA negative AA patients with SSc.

Previous studies investigated potential association of HLA DRB $1 * 08: 04$ with other rheumatic conditions like SLE (29) and RA (30). Reveille et al. detected no difference in frequency of HLA $D R B 1^{*} 08: 04$ between 88 AA patients with SLE or 88 unaffected AA controls. In another study by Hughes LB et al. no difference was reported in frequency of HLA $D R B 1 * 08: 04$ between 321 AA patients with RA and 564 unaffected individuals (30). Previously, we showed that HLA DRB $1 * 08: 04$ might be a susceptibility gene for SSc among AA (14); while the results of the current study demonstrated that the higher frequency of HLA $D R B 1 * 08: 04$ with SSc in AA patients is mainly driven by its strong association with AFA in this ethnic group. Furthermore, through the Binding Peptide Prediction Server for HLA $D R B 1 * 0804$, we identified four potential binding peptides from the human fibrillarin protein that could serve as potential binding sites within the antigen binding groove. The large effect sizes (table 2 and supplement table 1) and predicted binding peptides should prompt more studies to investigate their potential causal and/or environmental relationship of these autoantibodies.

In this regard, an animal model for induction of AFA has been extensively studied and may provide clues to an environmental trigger in humans with AFA-positive SSc. Certain mouse strains possessing specific $\mathrm{H} 2$ (the murine counterpart for HLA) haplotypes develop a nonSSc autoimmune disease and high titer AFA following administration of mercuric chloride or silver nitrate (31-34). Of note, one study of urinary mercury levels in SSc patients noted higher levels in those with AFA. However, this observation did not maintain statistical significance following corrections (35). Interestingly, heavy metals have previously been noted to be highly concentrated in the nucleolus (36). It was noted by Pollard et al. (37) that most if not all of the SSc-specific autoantigens were at sometime during their life cycle localized to the nucleolus. Clearly, larger and more targeted studies of heavy metal and other environmental exposures in AFA-positive SSc patients, perhaps selected for the associated HLA-class II alleles (DRB $1 * 13: 02$ in Caucasians and $D R B 1 * 08: 04$ in AAs) and AFA negative SSc patients, as well as well-matched normal controls are warranted.

Confirming our previous findings (10), we showed that HLA $D Q B 1 * 03: 01$ has higher frequency among AFA positive AA patients compared to AA unaffected individuals. However, there is no difference between AFA positive and negative AA patients with SSc.

AA SSc patients are younger at SSc onset compared to other ethnic groups $(1 ; 2 ; 5 ; 28)$. Moreover, other studies have shown that SSc patients with AFA have younger age of onset $(2 ; 12 ; 13 ; 16 ; 17)$. In support of these findings, we further demonstrated that AA SSc patients with AFA had younger age of onset in comparison to AA patients without AFA. 
Higher Medsger peripheral vascular severity index and prevalence of digital ulcers in AA patients with AFA compared to those without AFA are novel findings. These findings were also present when we compared AFA vs. ATA positive AA patients with SSc. Previous studies have shown higher rate of digital ulcers among AA patients with SSc compared to Caucasians $(2 ; 4)$. Higher frequency of AFA in AAs might contribute to this finding. In another study, Steen et al noted higher frequency of digital ulcers in AFA positive patients (12). However, these findings were not stratified for ethnic background.

In agreement with previous studies reporting more severe GI involvement in AFA positive patients (regardless of ethnicity) $(10 ; 12)$, we observed a higher frequency of SSc-associated diarrhea in AFA positive AA patients. The higher frequency of lower GI tract involvement was more significant when AFA positive AA patients were compared to ATA positive patients. It is possible that AFA positive patients have more severe lower GI tract hypomotility and bacterial overgrowth that contribute to diarrhea.

Our results imply a less severe lung involvement among AFA positive AA patients with SSc, as assessed by lower Medsger lung severity index. The comparison of AFA vs. ATA positive AA scleroderma patients further demonstrated less severe lung involvement (higher FVC and DLCO \% predicted values and lower Medsger lung severity index). In agreement with our findings, in an ethnically homogenous cohort of Japanese patients with SSc, AFA positive patients had less severe lung involvement (17). While data from several multiethnic cohorts suggested a higher frequency of isolated PAH and/or pulmonary fibrosis in the SSc patients with AFA $(9 ; 10 ; 12 ; 13 ; 38)$, these comparisons were adjusted neither for ethnicity nor for other antibodies i.e. ATA, as potential confounders. Therefore, the higher frequency of lung fibrosis and PAH might be due to a sizeable AA population in AFA positive group and large number of Caucasian SSc patients in AFA negative group. More severe SSc-associated lung involvement in AA patients with SSc compared to other ethnic groups has been reported in several studies $(2 ; 18 ; 28 ; 39 ; 40)$.

Based on the current findings, AFA positive AA patients with SSc have a higher prevalence of pericarditis, compared to AFA negative as well as and ATA positive patients. This is in agreement with former studies indicating higher frequency of cardiac involvement in AFA positive $(10 ; 12)$ and AA patients with SSc (39).

Our study did not confirm previous reports of worse (13) or better (11) survival in AFA positive patients with SSc. The worse survival of AFA positive patients in a previous report (13) might be attributable to the confounding or modifying effects of ethnicity in studies that are not stratified by ethnicity. As AA ethnicity is associated with AFA positivity as well as poorer survival $(1 ; 10 ; 12 ; 13)$.

This study has some limitations. Although potentially important, the data on heavy metal exposure were not collected in the current study. The Medsger severity indices were only available in the patients from the longitudinal GENISOS cohort. High-resolution computed tomography scan (HRCT) and echocardiography were not performed on all patients, which might have lead to underreporting of pulmonary involvement, despite being the largest genetic study ever reported in AA patients with SSc, we might be underpowered to detect more subtle HLA associations with AFA in AA population.

In conclusion, AFA was the second most common anti-nuclear antibody in African Americans with SSc. Presence of AFA was strongly associated with the HLA DRB1*08:04 in the AA SSc patients. In addition, AA SSc patients with AFA had a younger age of disease onset, higher frequency of digital ulcer and pericarditis, more severe lower GI involvement, and less severe pulmonary involvement. Studies in the future should focus on environmental 
factors, such as heavy metal exposure, that may influence the B cell response and the immunopathology of the disease.

\section{Supplementary Material}

Refer to Web version on PubMed Central for supplementary material.

\section{Acknowledgments}

The current study is supported by grants the National Institute of Health (NIH) Center of Research Translation P50AR054144 (Arnett and Mayes); NIH Training grant 5T32-AR052283-03 (Reveille); NIH Family registry and DNA repository N01-AR0-2251 (Mayes); NIH-KL2RR024149-04 (Assassi); NIH- U01-AI090909-01 Studies of HLA Region Genomics in Systemic Sclerosis and Ankylosing Spondylitis (Zhou); the United States Army Medical Research And Material Command- PR064251 Candidate Gene Polymorphisms in Scleroderma: Defining Genetic Susceptibility Factors (Mayes); University Clinic Research Center Grants: M01-RR00073 (UTMB) and M01RR01346 (UT-HSC-SA); NIH Clinical and Translational Sciences Award UL1-RR024148 and TL1 RR024147 from the National Center for Research Resources. However, the content is solely the responsibility of authors and does not represent the official views of the funding institutions.

\section{References}

1. Mayes MD, Lacey JV Jr, Beebe-Dimmer J, Gillespie BW, Cooper B, Laing TJ, et al. Prevalence, incidence, survival, and disease characteristics of systemic sclerosis in a large US population. Arthritis Rheum. 2003 Aug; 48(8):2246-55. [PubMed: 12905479]

2. Nietert PJ, Mitchell HC, Bolster MB, Shaftman SR, Tilley BC, Silver RM. Racial variation in clinical and immunological manifestations of systemic sclerosis. J Rheumatol. 2006 Feb; 33(2): 263-8. [PubMed: 16465656]

3. Assassi S, Del JD, Sutter K, McNearney TA, Reveille JD, Karnavas A, et al. Clinical and genetic factors predictive of mortality in early systemic sclerosis. Arthritis Rheum. 2009 Oct 15; 61(10): 1403-11. [PubMed: 19790132]

4. Beall AD, Nietert PJ, Taylor MH, Mitchell HC, Shaftman SR, Silver RM, et al. Ethnic disparities among patients with pulmonary hypertension associated with systemic sclerosis. J Rheumatol. 2007 Jun; 34(6):1277-82. [PubMed: 17516621]

5. Reveille JD, Fischbach M, McNearney T, Friedman AW, Aguilar MB, Lisse J, et al. Systemic sclerosis in 3 US ethnic groups: a comparison of clinical, sociodemographic, serologic, and immunogenetic determinants. Semin Arthritis Rheum. 2001 Apr; 30(5):332-46. [PubMed: 11303306]

6. Ochs RL, Lischwe MA, Spohn WH, Busch H. Fibrillarin: a new protein of the nucleolus identified by autoimmune sera. Biol Cell. 1985; 54(2):123-33. [PubMed: 2933102]

7. Ceribelli A, Cavazzana I, Franceschini F, Airo P, Tincani A, Cattaneo R, et al. Anti-Th/To Are Common Antinucleolar Autoantibodies in Italian Patients with Scleroderma. J Rheumatol. 2010 Aug 3.

8. Kuwana M, Okano Y, Kaburaki J, Tojo T, Medsger TA Jr. Racial differences in the distribution of systemic sclerosis-related serum antinuclear antibodies. Arthritis Rheum. 1994 Jun; 37(6):902-6. [PubMed: 8003063]

9. Okano Y, Steen VD, Medsger TA Jr. Autoantibody to U3 nucleolar ribonucleoprotein (fibrillarin) in patients with systemic sclerosis. Arthritis Rheum. 1992 Jan; 35(1):95-100. [PubMed: 1731817]

10. Arnett FC, Reveille JD, Goldstein R, Pollard KM, Leaird K, Smith EA, et al. Autoantibodies to fibrillarin in systemic sclerosis (scleroderma). An immunogenetic, serologic, and clinical analysis. Arthritis Rheum. 1996 Jul; 39(7):1151-60. [PubMed: 8670324]

11. Tormey VJ, Bunn CC, Denton CP, Black CM. Anti-fibrillarin antibodies in systemic sclerosis. Rheumatology (Oxford). 2001 Oct; 40(10):1157-62. [PubMed: 11600746]

12. Steen VD. Autoantibodies in systemic sclerosis. Semin Arthritis Rheum. 2005 Aug; 35(1):35-42. [PubMed: 16084222]

13. Aggarwal R, Lucas M, Fertig N, Oddis CV, Medsger TA Jr. Anti-U3 RNP autoantibodies in systemic sclerosis. Arthritis Rheum. 2009 Apr; 60(4):1112-8. [PubMed: 19333934] 
14. Arnett FC, Gourh P, Shete S, Ahn CW, Honey RE, Agarwal SK, et al. Major histocompatibility complex (MHC) class II alleles, haplotypes and epitopes which confer susceptibility or protection in systemic sclerosis: analyses in 1300 Caucasian, African-American and Hispanic cases and 1000 controls. Ann Rheum Dis. 2010 May; 69(5):822-7. [PubMed: 19596691]

15. Satoh M, Akizuki M, Kuwana M, Mimori T, Yamagata H, Yoshida S, et al. Genetic and immunological differences between Japanese patients with diffuse scleroderma and limited scleroderma. J Rheumatol. 1994 Jan; 21(1):111-4. [PubMed: 8151564]

16. Reimer G, Steen VD, Penning CA, Medsger TA Jr, Tan EM. Correlates between autoantibodies to nucleolar antigens and clinical features in patients with systemic sclerosis (scleroderma). Arthritis Rheum. 1988 Apr; 31(4):525-32. [PubMed: 2451921]

17. Kuwana M, Kaburaki J, Okano Y, Tojo T, Homma M. Clinical and prognostic associations based on serum antinuclear antibodies in Japanese patients with systemic sclerosis. Arthritis Rheum. 1994 Jan; 37(1):75-83. [PubMed: 8129766]

18. Assassi S, Sharif R, Lasky RE, McNearney TA, Estrada YMR, Draeger H, et al. Predictors of interstitial lung disease in early systemic sclerosis: a prospective longitudinal study of the GENISOS cohort. Arthritis Res Ther. 2010 Sep 2.12(5):R166. [PubMed: 20813056]

19. Mayes MD. The establishment and utility of a population-based registry to understand the epidemiology of systemic sclerosis. Curr Rheumatol Rep. 2000 Dec; 2(6):512-6. [PubMed: 11123106]

20. Preliminary criteria for the classification of systemic sclerosis (scleroderma). Subcommittee for scleroderma criteria of the American Rheumatism Association Diagnostic and Therapeutic Criteria Committee. Arthritis Rheum. 1980 May; 23(5):581-90. [PubMed: 7378088]

21. Leroy EC, Black C, Fleischmajer R, Jablonska S, Krieg T, Medsger TA Jr, et al. Scleroderma (systemic sclerosis): classification, subsets and pathogenesis. J Rheumatol. 1988 Feb; 15(2):202-5. [PubMed: 3361530]

22. Olsen ML, Arnett FC, Reveille JD. Contrasting molecular patterns of MHC class II alleles associated with the anti-Sm and anti-RNP precipitin autoantibodies in systemic lupus erythematosus. Arthritis Rheum. 1993 Jan; 36(1):94-104. [PubMed: 7678744]

23. Sturniolo T, Bono E, Ding J, Raddrizzani L, Tuereci O, Sahin U, et al. Generation of tissuespecific and promiscuous HLA ligand databases using DNA microarrays and virtual HLA class II matrices. Nat Biotechnol. 1999 Jun; 17(6):555-61. [PubMed: 10385319]

24. Singh H, Raghava GP. ProPred: prediction of HLA-DR binding sites. Bioinformatics. 2001 Dec; 17(12):1236-7. [PubMed: 11751237]

25. Clements PJ, Lachenbruch PA, Seibold JR, Zee B, Steen VD, Brennan P, et al. Skin thickness score in systemic sclerosis: an assessment of inter observer variability in 3 independent studies. J Rheumatol. 1993 Nov; 20(11):1892-6. [PubMed: 8308774]

26. Steen VD, Medsger TA Jr, Rodnan GP. D-Penicillamine therapy in progressive systemic sclerosis (scleroderma): a retrospective analysis. Ann Intern Med. 1982 Nov; 97(5):652-9. [PubMed: 7137731]

27. Reveille JD, Durban E, Goldstein R, Moreda R, Arnett FC. Racial differences in the frequencies of scleroderma-related autoantibodies. Arthritis Rheum. 1992 Feb; 35(2):216-8. [PubMed: 1734910]

28. Reveille JD. Ethnicity and race and systemic sclerosis: how it affects susceptibility, severity, antibody genetics, and clinical manifestations. Curr Rheumatol Rep. 2003 Apr; 5(2):160-7. [PubMed: 12628048]

29. Reveille JD, Moulds JM, Ahn C, Friedman AW, Baethge B, Roseman J, et al. Systemic lupus erythematosus in three ethnic groups: I. The effects of HLA class II, C4, and CR1 alleles, socioeconomic factors, and ethnicity at disease onset. LUMINA Study Group. Lupus in minority populations, nature versus nurture. Arthritis Rheum. 1998 Jul; 41(7):1161-72. [PubMed: 9663471]

30. Hughes LB, Morrison D, Kelley JM, Padilla MA, Vaughan LK, Westfall AO, et al. The HLADRB1 shared epitope is associated with susceptibility to rheumatoid arthritis in African Americans through European genetic admixture. Arthritis Rheum. 2008 Feb; 58(2):349-58. [PubMed: 18240241]

31. Hultman P, Enestrom S, Pollard KM, Tan EM. Anti-fibrillarin autoantibodies in mercury-treated mice. Clin Exp Immunol. 1989 Dec; 78(3):470-7. [PubMed: 2612058] 
32. Chen M, Rockel T, Steinweger G, Hemmerich P, Risch J, von MA. Subcellular recruitment of fibrillarin to nucleoplasmic proteasomes: implications for processing of a nucleolar autoantigen. Mol Biol Cell. 2002 Oct; 13(10):3576-87. [PubMed: 12388758]

33. Havarinasab S, Pollard KM, Hultman P. Gold- and silver-induced murine autoimmunity-requirement for cytokines and CD28 in murine heavy metal-induced autoimmunity. Clin Exp Immunol. 2009 Mar; 155(3):567-76. [PubMed: 19077085]

34. Pollard KM, Hultman P, Kono DH. Toxicology of autoimmune diseases. Chem Res Toxicol. 2010 Mar 15; 23(3):455-66. [PubMed: 20078109]

35. Arnett FC, Fritzler MJ, Ahn C, Holian A. Urinary mercury levels in patients with autoantibodies to U3-RNP (fibrillarin). J Rheumatol. 2000 Feb; 27(2):405-10. [PubMed: 10685806]

36. Rosen A, Casciola-Rosen L, Wigley F. Role of metal-catalyzed oxidation reactions in the early pathogenesis of scleroderma. Curr Opin Rheumatol. 1997 Nov; 9(6):538-43. [PubMed: 9375283]

37. Pollard KM, Reimer G, Tan EM. Autoantibodies in scleroderma. Clin Exp Rheumatol. 1989 Sep; 7(Suppl 3):S57-S62. [PubMed: 2691161]

38. Sacks DG, Okano Y, Steen VD, Curtiss E, Shapiro LS, Medsger TA Jr. Isolated pulmonary hypertension in systemic sclerosis with diffuse cutaneous involvement: association with serum anti-U3RNP antibody. J Rheumatol. 1996 Apr; 23(4):639-42. [PubMed: 8730118]

39. Laing TJ, Gillespie BW, Toth MB, Mayes MD, Gallavan RH Jr, Burns CJ, et al. Racial differences in scleroderma among women in Michigan. Arthritis Rheum. 1997 Apr; 40(4):734-42. [PubMed: 9125258]

40. McNearney TA, Reveille JD, Fischbach M, Friedman AW, Lisse JR, Goel N, et al. Pulmonary involvement in systemic sclerosis: associations with genetic, serologic, sociodemographic, and behavioral factors. Arthritis Rheum. 2007 Mar 15; 57(2):318-26. [PubMed: 17330281] 


\section{Table 1}

Study population $(n=278)$

\begin{tabular}{|c|c|}
\hline Age, mean $( \pm \mathrm{SD})$, yrs & $46.9(13.6)$ \\
\hline Gender, female, n (\%) & $237(85.3)$ \\
\hline Cutaneous involvement, diffuse, $\mathrm{n}(\%)$ & $171(61.5)$ \\
\hline Disease duration, mean $( \pm \mathrm{SD})$, yrs & $6.0(6.5)$ \\
\hline MRSS, mean $( \pm$ SD) & $17.3(12.3)$ \\
\hline Deceased patients, $\mathrm{n}(\%)$ & $83(29.7)$ \\
\hline Survival time (from time of enrollment), mean $( \pm \mathrm{SD})$, yrs & $5.8(5.0)$ \\
\hline \multicolumn{2}{|l|}{ Autoantibody profile, $\%$} \\
\hline$A N A^{*}$ & 93.1 \\
\hline$A C A^{* *}$ & 6.2 \\
\hline$A T A^{\dagger}$ & 21.8 \\
\hline$A N o A^{+}$ & 44.5 \\
\hline$A F A^{\beta}$ & 18.5 \\
\hline Pol III ${ }^{\gamma}$ & 15.4 \\
\hline$U 1-R N P^{4}$ & 12.2 \\
\hline$P M / S c l^{\S}$ & 3.2 \\
\hline $\operatorname{Ro}^{\Phi}$ & 9.3 \\
\hline \multicolumn{2}{|l|}{ * ANA, anti-nuclear antibodies; } \\
\hline \multicolumn{2}{|l|}{ ** ACA, anti-centromere antibodies; } \\
\hline \multicolumn{2}{|l|}{${ }^{\dagger}$ ATA, anti-topoisomerase I antibodies; } \\
\hline \multicolumn{2}{|l|}{ ANoA, anti-nucleolar antibodies; } \\
\hline \multicolumn{2}{|l|}{${ }^{\beta} \mathrm{AFA}$, anti-fibrillarin antibodies; } \\
\hline \multicolumn{2}{|l|}{$\gamma_{\text {Pol III, RNA polymerase III; }}$} \\
\hline \multicolumn{2}{|l|}{${ }^{\Delta}$ U1-RNP, U1-ribonucleoprotein; } \\
\hline$\S_{\mathrm{PM} / \mathrm{Scl} \text {, polymyositis-scleroderma antigen; }}$ & \\
\hline
\end{tabular}




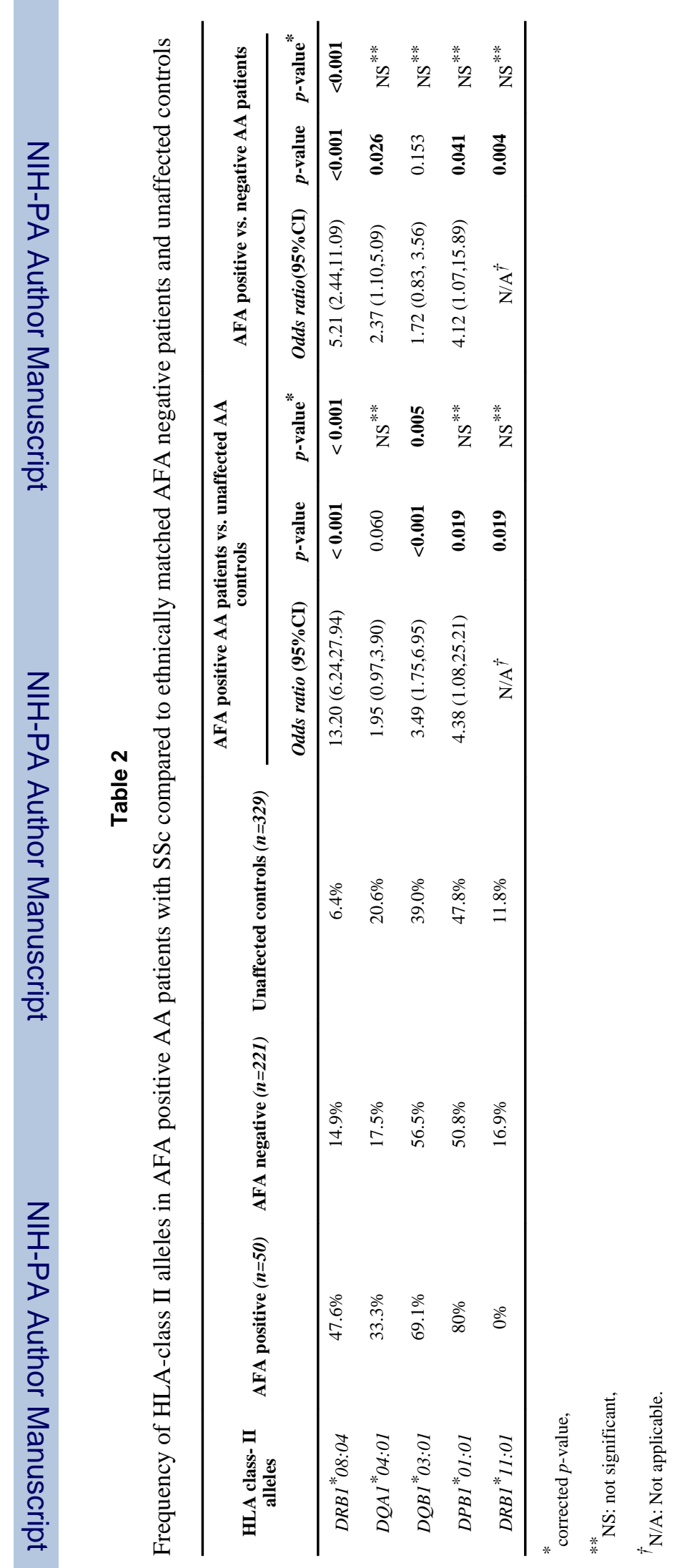

J Rheumatol. Author manuscript; available in PMC 2012 August 1. 
Table 3

Clinical manifestations of African American patients with AFA compared to those without AFA (adjusted for disease duration)

\begin{tabular}{|c|c|c|c|c|}
\hline & AFA positive $(n=50)$ & AFA negative ( $n=221)$ & Odds ratio $(95 \% \mathrm{CI})$ & $p$-value \\
\hline Age $^{*}$, mean $( \pm S D), y r$ & $41.7(13.31)$ & $47.9(13.25)$ & $-6.29(-10.59,-1.99)^{* *}$ & 0.004 \\
\hline Gender ${ }^{*}$, Female, $\%$ & 86.0 & 84.6 & $0.89(0.31,2.24)$ & 0.806 \\
\hline Cutaneous involvement ${ }^{*}$, diffuse, $\%$ & 36.0 & 39.5 & $0.86(0.43,1.69)$ & 0.642 \\
\hline Disease duration at enrollment ${ }^{*}$ & $5.34(5.14)$ & $6.28(6.85)$ & $-0.93(-3.51,1.64)^{* *}$ & 0.475 \\
\hline Deceased, \% & 30.0 & 29.5 & $0.99(0.47,2.03)$ & 0.892 \\
\hline $\mathrm{MRSS}^{\dagger}$, mean $( \pm \mathrm{SD})$ & $14.31(7.62)$ & $17.83(13.50)$ & $-2.55(-9.66,4.54)^{* *}$ & 0.476 \\
\hline Digital ulcer, \% & 79.3 & 53.8 & $3.31(1.27,8.62)$ & 0.014 \\
\hline Dysphagia, \% & 60.0 & 54.8 & $1.24(0.49,3.19)$ & 0.619 \\
\hline Diarrhea, \% & 53.9 & 19.2 & $4.84(1.29,18.13)$ & 0.019 \\
\hline Pericarditis, \% & 28.2 & 12.8 & $2.45(0.86,6.93)$ & 0.092 \\
\hline $\mathrm{PAH}^{+}, \%$ & 10.0 & 22.4 & $0.38(0.09,1.19)$ & 0.081 \\
\hline SSc interstitial lung fibrosis, $\%$ & 28.2 & 47.3 & $0.65(0.28,1.52)$ & 0.322 \\
\hline FVC $\%$ predicted, mean $( \pm \mathrm{SD})$ & $77.8(17.7)$ & $72.9(23.2)$ & $6.08(-4.54,16.69)^{* *}$ & 0.259 \\
\hline DLCO $\%$ predicted, mean $( \pm \mathrm{SD})$ & $67.7(17.2)$ & $57.9(24.6)$ & $9.45(-2.24,21.14)^{* *}$ & 0.112 \\
\hline SSc renal crisis, $\%$ & 10.5 & 8.4 & $1.28(0.28,4.54)$ & 0.921 \\
\hline Myositis or muscle weakness, $\%$ & 30.0 & 40.6 & $1.05(0.21,5.28)$ & 0.953 \\
\hline Elevated serum CK & 28.6 & 30.9 & $0.88(0.31,2.54)$ & 0.817 \\
\hline Arthritis, $\%$ & 21.9 & 31.0 & $0.53(0.13,2.13)$ & 0.370 \\
\hline Joint contracture & 22.9 & 21.4 & $1.44(0.56,3.72)$ & 0.447 \\
\hline Sicca $\S_{\text {symptoms }}$ & 20.0 & 28.1 & $0.64(0.11,3.69)$ & 0.621 \\
\hline \multicolumn{5}{|l|}{ Medsger Severity Index, mean $( \pm S D)$} \\
\hline General & 0.6 & 0.5 & $0.06(-0.53,0.64)^{* *}$ & 0.838 \\
\hline Peripheral vascular & 2.2 & 1.4 & $0.79(0.27,1.30)^{* *}$ & 0.003 \\
\hline Skin & 1.5 & 1.7 & $-0.27(-0.84,0.31)^{* *}$ & 0.361 \\
\hline Joint & 0.8 & 0.9 & $-0.09(-0.92,0.73)^{* *}$ & 0.813 \\
\hline Muscle & 0.2 & 0.3 & $-0.10(-0.41,0.21)^{* *}$ & 0.521 \\
\hline GI Tract & 0.6 & 0.6 & $0.02(-0.43,0.39)^{* *}$ & 0.935 \\
\hline Lung & 1.1 & 1.9 & $-0.82(-1.50,-0.14)^{* *}$ & 0.019 \\
\hline Heart & 0.5 & 0.3 & $0.16(-0.27,0.58)^{* *}$ & 0.457 \\
\hline Kidney & 0.1 & 0.3 & $0.19(-0.68,0.29)^{* *}$ & 0.441 \\
\hline $\begin{array}{l}\text { These comparisons were not adjusted } \\
\text { numbers indicate the mean difference }\end{array}$ & . & 2 & ed for comparisons, accord & \\
\hline
\end{tabular}

J Rheumatol. Author manuscript; available in PMC 2012 August 1. 


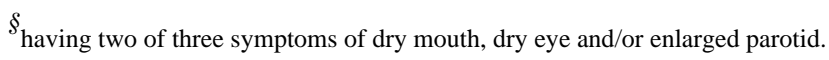


Table 4

Cox proportional hazards regression model analysis of AA patients with systemic sclerosis (SSc)

\begin{tabular}{lcc}
\hline & Hazard Ratio $(\mathbf{9 5 \%}$ Confidence interval) & p-value \\
\hline AFA & $0.80(0.41,1.53)$ & 0.493 \\
AFA Vs. ATA & $0.84(0.42,1.69)$ & 0.623 \\
HLA-DRB ${ }^{*} 08: 04$ & $1.00(0.55,1.83)$ & 0.996 \\
HLA- $D Q A 1 * 04: 01$ & $1.50(0.84,2.68)$ & 0.170 \\
HLA-DQB $1^{*} 03: 01$ & $0.85(0.51,1.41)$ & 0.520 \\
HLA- $D Q B 1 * 01: 01$ & $1.13(0.49,2.60)$ & 0.766 \\
HLA-DRB $1 * 11: 01$ & $1.17(0.57,2.39)$ & 0.671 \\
\hline
\end{tabular}

